\title{
Quantum Gravity Strategy for the Production of Dark Matter Using Cavitation by Minimum Entropy ${ }^{\dagger}$
}

\author{
Edward Jiménez ${ }^{1, *}$ and Esteban E. Jimenez ${ }^{2}$ \\ 1 Faculty of Chemical Engineering, Central University of Ecuador (UCE), Gerónimo Leiton S/N y Gatto \\ Sobral. Telf (5932) 2524766, Quito 170521, Ecuador \\ 2 Institut National des Sciences et Techniques Nucléaires, 91400 Saclay, France; estebanjimenezec@gmail.com \\ * Correspondence: ehjimenez@uce.edu.ec \\ + Presented at the 5th International Electronic Conference on Entropy and Its Applications, 18-30 November \\ 2019; Available online: https:/ / ecea-5.sciforum.net/.
}

Published: 17 November 2019

\begin{abstract}
The minimum entropy is responsible for the formation of dark matter bubbles in a black hole, while the variation in the density of dark matter allows these bubbles to leave the event horizon. Some experimental evidence supports the dark matter production model in the inner vicinity of the border of a black hole. The principle of minima entropy explains how cavitation occurs on the event horizon, which in turn complies with the Navier-Stokes 3D equations. Moreover, current works in an axiomatic way show that in the event horizon Einstein's equations are equivalent to Navier-Stokes' equations. Thus, The solutions of Einstein combined with the boundary conditions establish a one-to-one correspondence with solutions of incompressible Navier-Stokes and in the near-horizon limit it provides a precise mathematical sense in which horizons are always incompressible fluids. It is also essential to understand that Cavitation by minimum entropy is the production of dark matter bubbles, by variation of the pressure inside or on the horizon of a black hole, in general $\Delta p=p_{n+1}-p_{n}=\left(\frac{\sigma_{n}}{\sigma_{n+1}}-1\right) p_{n}$ or in particular $\Delta p=-(1-P) p_{0}$, where $\frac{\partial P}{\partial t}=\frac{\Delta p}{\rho_{0}} P$. Finally, fluctuations in the density of dark matter can facilitate its escape from a black hole, if and only if there is previously dark matter produced by cavitation inside or on the horizon of a black hole and also $\rho_{D M}<\rho_{B}$.
\end{abstract}

\section{Introduction}

Capturing the first direct image of a black hole using Event Horizon Telescope (EHT), was a revolution in Astrophysics in 2019, and the team of researchers has received the respective credits and has opened the possibility that these black holes will be generated inside dark matter [1]. "We have been studying black holes for so long that sometimes it is easy to forget that none of us have ever actually seen one," said France Córdova, the director of the National Science Foundation, at a press conference in Washington, DC.

Doeleman and hundreds of other scientists used data from eight telescopes located around the world, creating an "eye" the size of the Earth integral enough to look inside a galaxy in the Virgo cluster, about 55 million light years of planet earth. After many months of continuous analysis, they discovered that the ring, which marked the orbit that light takes around the black hole, was real.

Alexander Kashlinsky, a NASA-Goddard scientist, suggests a possible link between primordial black holes and dark matter [1,2].

Dark matter is an invisible and undetectable substance directly that makes up most of the material universe. A new vision proposes that dark matter is made of black holes formed during the first second of the existence of our universe, known as primordial black holes [3-5]. Currently, a NASA scientist Goddard in Greenbelt, Maryland, suggests that this interpretation aligns with our knowledge 
of the cosmic x-ray and infrared background rays and can explain the unexpectedly high masses of newly detected fused black holes. In addition, this cosmic infrared background (CIB) has a similar structure in other parts of the universe.

Could dark matter be made of primordial black holes, as numerous as the stars? It is possible that the discovery of gravitational waves suggested that in the cosmos abounds with unexpectedly heavy black holes and of this manner we can explain the universe's missing mass $[5,6]$.

In the proper way Bredberg et al. synthesize the respective theorems From Einstein to Navier-Stokes, we complement the chain of implications from Navier-Stokes to dark matter production by minimum entropy.

The solutions of Einstein combined with the boundary conditions we impose correspondence one-to-one with solutions of incompressible Navier-Stokes." and "Our near-horizon limit provides a precise mathematical sense in which horizons are incompressible fluids" [6,7].

It has been shown that gravitational systems manifest behaviors according to Navier-Stokes when we study the dynamics of the region near an event horizon (macroscopic or quantum level). Thus, it is possible to find non-linear deformations of Einstein's solutions [8-10].

\section{Model}

Using the cavitation and Navier-Stokes 3D equations we can explain the production of dark matter in black holes and especially in the event horizon, see Figure 1. This risky conjecture has the support of the advances made in the first photo of a black hole and its correspondence with Einstein's gravitation equations. In addition, it is categorically demonstrated that Einstein's equations not only relate to Navier-Stokes' equations but are equivalent in the event horizon [9-11]. Bredberg et al. (2012) probes the respective theorems from Einstein to Navier-Stokes as follows. "The near-horizon expansion in gravity is shown to be mathematically equivalent to the hydrodynamic expansion in fluid dynamics, and the Einstein equation reduces to the incompressible Navier-Stokes equation."

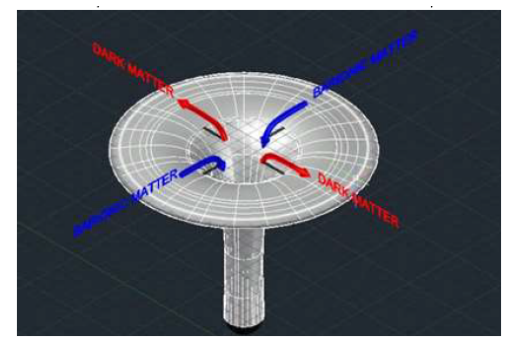

Figure 1. Dark matter production inside a black hole.

\section{Cavitation}

Cavitation in the inner neighborhood of the event horizon can be fully explained by the Navier-Stokes 3D equations, which in turn biunivocally represent Einstein's gravitational equations. The formation of dark matter bubbles facilitates their expulsion by the black hole as a whole. While a black hole eats barionic matter it expels dark matter.

Dimensional Analysis.

We define the respective dimensional units of each one of variables and physical constants that appear in the solution of the Navier-Stokes 3D equation.

Kinematic viscosity $\quad v=\frac{\eta}{\rho_{0}},\left[\frac{\mathrm{m}^{2}}{\mathrm{~s}}\right]$

Dynamic viscosity $\quad \eta$, [pa.s], where [pa] represents pascal pressure unit.

Initial pressure of out of equilibrium. $p_{0},[\mathrm{pa}]$

Fluid density $\rho_{0},\left[\frac{\mathrm{kg}}{\mathrm{m}^{3}}\right]$, where $\mathrm{kg}$ is kilogram and $\mathrm{m}^{3}$ cubic meters.

Logistic probability function, $P(x, y, z, t)=\frac{1}{1+e^{k t-\mu r}}$, it is a real number $0 \leq P \leq 1$. 
Equilibrium condition $\quad, r=\frac{k}{\mu} t=\frac{p_{0}}{2 \rho_{0} v \mu} t=\left|\mathbf{u}_{e}\right| t,[\mathrm{~m}]$.

Fluid velocity in equilibrium, $\left|\mathbf{u}_{e}\right|,[\mathrm{m} / \mathrm{s}]$.

Fluid field velocity out of equilibrium, $\mathbf{u}=-2 v \mu(1-P) \nabla r$. [m/s].

Position, $r=\left(x^{2}+y^{2}+z^{2}\right)^{1 / 2},[\mathrm{~m}]$.

Attenuation coefficient, $\mu,[1 / \mathrm{m}]$.

Growth coefficient, $k=\frac{p_{0}}{2 \rho_{0} v},[1 / \mathrm{s}]$.

Concentration $C=C_{0} \frac{1-P}{P}$.

The fluid speed needs to be defined as $\mathbf{u}=-2 v \frac{\nabla P}{P}$, where $P(x, y, z, t)$ is the logistic probability function $P(x, y, x, t)=\frac{1}{1+e^{k t-\mu r}}, r=\left(x^{2}+y^{2}+z^{2}\right)^{1 / 2}$ defined in $\left((x, y, z) \in \mathbb{R}^{3}, t \geq 0\right)$. This $P$ is the general solution of the Navier-Stokes 3D equations, which satisfies the conditions in Equations (1) and (2), allowing us to analyze the dynamics of an incompressible fluid.

$$
\frac{\partial \mathbf{u}}{\partial t}+(\mathbf{u} . \nabla) \mathbf{u}=-\frac{\nabla p}{\rho_{0}} \quad\left((x, y, z) \in \mathbb{R}^{3}, t \geq 0\right)
$$

Where, $\mathbf{u} \in \mathbb{R}^{3}$ an known velocity vector, $\rho_{0}$ constant density of fluid and pressure $p=p_{0} P \in \mathbb{R}$. follows.

With speed and pressure dependent on $r$ and $t$. We write the condition of incompressibility as

$$
\nabla \cdot \mathbf{u}=0 \quad\left((x, y, z) \in \mathbb{R}^{3}, t \geq 0\right)
$$

Theorem 1. Navier-Stokes $3 D$ solutions. The velocity of the fluid given by: $\mathbf{u}=-2 v \frac{\nabla P}{P}$, where $P(x, y, z, t)$ is the logistic probability function $P(x, y, x, t)=\frac{1}{1+e^{k t-\mu\left(x^{2}+y^{2}+z^{2}\right)^{1 / 2}}}$, defined in $\left((x, y, z) \in \mathbb{R}^{3}, t \geq 0\right)$ is the general solution of the Navier-Stokes equations, which satisfies conditions in Equations (1) and (2).

Proof. Firstly, we will make the equivalence $\mathbf{u}=\nabla \theta$ and replace it in Equation (1). Taking into account that $\nabla \theta$ is irrotational, $\nabla \times \nabla \theta=0$, we have.

$$
(\mathbf{u} . \nabla) \mathbf{u}=(\nabla \theta \cdot \nabla) \nabla \theta=\frac{1}{2} \nabla(\nabla \theta \cdot \nabla \theta)-\nabla \theta \times(\nabla \times \nabla \theta)=\frac{1}{2} \nabla(\nabla \theta . \nabla \theta),
$$

We can write,

$$
\nabla\left(\frac{\partial \theta}{\partial t}+\frac{1}{2}(\nabla \theta \cdot \nabla \theta)\right)=\nabla(-p)
$$

It is equivalent to,

$$
\frac{\partial \theta}{\partial t}+\frac{1}{2}(\nabla \theta \cdot \nabla \theta)=-\frac{\Delta p}{\rho_{0}}
$$

where $\Delta p$ is the difference between the actual pressure $p$ and certain reference pressure $p_{0}$. Now, replacing $\theta=-2 v \ln (P)$, Navier-Stokes equation becomes.

$$
\frac{\partial P}{\partial t}=\frac{\Delta p}{\rho_{0}} P
$$

The external force is zero, so that there is only a constant force $F$ due to the variation of the pressure on a cross section $\sigma$. Where $\sigma$ is the total cross section of all events that occurs in cavitation and bubbles formation including: loss of energy, absorption and creation of shock waves.

$$
\begin{aligned}
F & =p \sigma_{2}=p_{0} \sigma_{1} \\
\Delta p & =p-p_{0}=\left(\frac{\sigma_{1}}{\sigma_{2}}-1\right) p_{0}=-(1-P) p_{0}
\end{aligned}
$$

putting (3) in (4) we have

$$
\frac{\partial P}{\partial t}=-\mu k(1-P) P
$$


In order to verify Equation (2), $\nabla \cdot \mathbf{u}=0$, we need to obtain $\nabla r=\left(\frac{x}{r}, \frac{y}{r}, \frac{z}{r}\right), \nabla^{2} r=\nabla \cdot \nabla r$ $=\frac{\left(y^{2}+z^{2}\right)+\left(x^{2}+z^{2}\right)+\left(x^{2}+y^{2}\right)}{\left(x^{2}+y^{2}+z^{2}\right)^{3 / 2}}=\frac{2}{r}$.

$$
\nabla \cdot u=-2 v \nabla \cdot \frac{\nabla P}{P}=-2 v \mu \nabla((1-P) \nabla r)
$$

Replacing the respective values for the terms: $\nabla^{2} P$ and $|\nabla P|^{2}$ of Equation (6). The Laplacian of $P$ can be written as follows.

$$
\begin{aligned}
\nabla^{2} P & =\mu(1-2 P) \nabla P . \nabla r+\mu\left(P-P^{2}\right) \nabla^{2} r \\
& =\mu^{2}(1-2 P)\left(P-P^{2}\right)|\nabla r|^{2}+\mu\left(P-P^{2}\right) \nabla^{2} r \\
& =\mu^{2}(1-2 P)\left(P-P^{2}\right)+\mu\left(P-P^{2}\right) \frac{2}{r}
\end{aligned}
$$

Using gradient $\nabla P=\mu\left(P-P^{2}\right) \nabla r$, modulus $|\nabla P|^{2}=\mu^{2}\left(P-P^{2}\right)^{2}|\nabla r|^{2}$ and $\nabla^{2} P$ in Equation (7).

$$
\left[\frac{\nabla^{2} P}{P}-\frac{|\nabla P|^{2}}{P^{2}}\right]=0
$$

Replacing Equations (6) and (7) in Equation (10), we obtain the main result of the Navier-Stokes equations, this solution represents a fixed point of an implicit function $f(t, r)$ where $f(t, r)=P-\frac{2}{\mu r}=$ 0 .

$$
P=\frac{1}{1+e^{k t-\mu\left(x^{2}+y^{2}+z^{2}\right)^{1 / 2}}}=\frac{2}{\mu\left(x^{2}+y^{2}+z^{2}\right)^{1 / 2}} \quad\left((x, y, z) \in \mathbb{R}^{3}, t \geq 0\right)
$$

Placing the parameters $k$ in function of variables of the fluid mechanics we have.

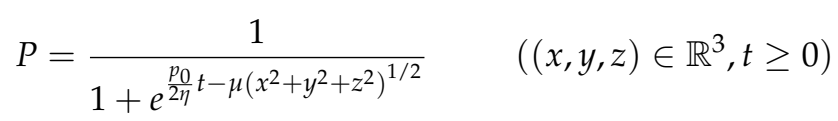

Theorem 2. Cavitation by minimum entropy is the production of dark matter bubbles, by variation of the pressure inside or on the horizon of a black hole, in a general way $\Delta p=p_{n+1}-p_{n}=\left(\frac{\sigma_{n}}{\sigma_{n+1}}-1\right) p_{n}$ or in particular way $\Delta p=-(1-P) p_{0}$, where $\frac{\partial P}{\partial t}=\frac{\Delta p}{\rho_{0}} P$ according to Equation (3)

Proof. By generalizing Equation (4), we can analyze the evolution of pressure and its intrinsic relationship with the effective section $\sigma_{n}$, a fundamental element of the solution of the Navier-Stokes $3 \mathrm{D}$ equations. In this sense, we know that the forces, in a physically infinitesimal or microscopic volume are conserved, therefore, we write $F=p_{n+1} \sigma_{n+1}=p_{n} \sigma_{n}$ from here we apply the condition of cavitation in which the pressure of the dark matter $p_{n+1}$ is greater than the pressure of the barionic matter $p_{n}$, that is $p_{n+1}<p_{n}$,or $\Delta p=p_{n+1}-p_{n}=\left(\frac{\sigma_{n}}{\sigma_{n+1}}-1\right) p_{n}$, That proves the theorem. In a particular way $p_{1}<p_{0}$, where $p_{1}$ represents the pressure of the dark matter and $p_{0}$ indicates the pressure of the baryonic matter. If we get the force conservation variable, then we have the pressure variation:

$$
\begin{aligned}
\Delta p & =p_{1}-p_{0}=\left(\frac{\sigma_{0}}{\sigma_{1}}-1\right) p_{0}=-(1-P) p_{0} \\
P & =\frac{1}{1+e^{k t-\mu\left(x^{2}+y^{2}+z^{2}\right)^{1 / 2}}}
\end{aligned}
$$

This last equation is the explicit way of solving the Navier-Stokes 3D equations, which depends on time and space. 
Proof. The mathematical proof must correspond to physical reality, as follows: to produce cavitation the only alternative is that at least it is fulfilled that $p_{1}<p_{0}$ at the beginning of the process, or on the event horizon.

Proof. Finally, this demonstration is consistent even if in the volume of analysis, naturally created vorticity phenomena occur as in a black hole or artificially as in ship propellers. Cavitation produces pressure variations and therefore can create dark matter.

Theorem 3. Fluctuations in the density of dark matter can facilitate its escape from a black hole, if and only if there is previously dark matter produced by cavitation inside or on the horizon of a black hole and also $\rho_{D M}<\rho_{B}$.

Proof. In order to meet the requirements of cavitation in the event horizon of a black hole we need to study some experimental evidence and the reference values of density, pressure and temperature of dark matter and barionic matter, respectively. Let $\rho_{D M}$, the density of dark matter and $\rho_{B}$, the density of baryonic matter, where randomly exist at some point: $\rho_{D M}<\rho_{B}$.

The black hole temperature is of the order of $10^{-4} \mathrm{~K}$. This allows Bose-Einstein condensates to form with extremely small densities of both dark matter and barionic matter.

The very high pressures inside the black hole, due to the intense gravitational force together with the non-linear instabilities of pressures of barionic matter and dark matter make it possible that at some points, at least in the vicinity of the event horizon, the density of the dark matter $\rho_{D M}$ is less than the density of the barionic matter $\rho_{D M}<\rho_{B}$ promoting the escape of dark matter if and only if it already exists inside or at the border of a black hole

Theorem 4. An action on bubble surface produces a reaction in the volume and vice versa.

Proof. The volume and the bubble surface are connected through the Gaussian divergence theorem and the Navier-Stokes equations. For an incompressible fluid compost of dark matter or barionic matter, whose velocity field $\vec{u}(x, y, z)$ is given, $\vec{\nabla} \cdot \vec{u}=0$ is fulfilled. Logically, the integral of this term remains zero, that is:

$$
\iiint \vec{\nabla} \cdot \vec{u} d x d y d z=0
$$

Writing the divergence theorem. $\iint \vec{u} \cdot \vec{n} d S=\iiint \vec{\nabla} \cdot \vec{u} d x d y d z=0$, the first term must be equal to zero, that is:

$$
\iint \vec{u} \cdot \vec{n} d S=\iint\|\vec{u}\|\|\vec{n}\| \cos (\alpha) d S=0 \rightarrow \alpha=\frac{\pi}{2}
$$

$\vec{n}$ is perpendicular to the surface of the sphere. In this way, the equation of the outer sphere corresponding to the surface is: $x^{2}+y^{2}+z^{2}=1.2 A^{1 / 3}$. Within the bubble there are layers of dark matter that move in spherical trajectories.

Acknowledgments: We would like to acknowledge the teachers and students of the Chemical Engineering Faculty of the Central University of Ecuador, especially to the GIIP Group, the participants of the congress 3rd Edition of International conference on Advanced Spectroscopy, Crystallography and Applications in Modern Chemistry during 4-5 June 2018 London, UK, and finally to the participants of the congress CIPANP 2018- "Thirteenth Conference on the Intersections of Particle and Nuclear Physics" 2018, Berkeley, USA.

\section{References}

1. Doeleman. First Ever Real Image of a Black Hole Revealed. 2019. Available online: https://www. newscientist.com/article/2199330 (accessed on 1 April 2020). 
2. De Nova, J.R.; Golubkov, K.; Kolobov, V.I.; Steinhauer, J. Observation of thermal Hawking radiation and its temperature in an analogue black hole. Nature 2019, 569, 688. doi:10.1038/s41586-019-1241-0.

3. Kashlinsky, A.; Ligo, G. Wave Detection, Primordial Black Holes, and the Near-Ir Cosmic Infrared Background Anisotropies. Astrophys. J. Lett. 2016 , 823, L25.

4. Hawking, S.W.; Israel, W. General Relativity: An Einstein Centenary Survey; Cambridge University Press: Cambridge, UK, 1979; p. 98, ISBN 978-0-521-22285-3.

5. Niikura, H.; Takada, M.; Yasuda, N.; Lupton, R.H.; Sumi, T.; More, S.; Kurita, T.; Sugiyama, S.; More, A.; Oguri, M.; et al. Microlensing constraints on primordial black holes with Subaru/HSC Andromeda observations. Nat. Astron. 2019, 3, 524-534.

6. Bogdan, A.; Goulding, A.D. Connecting Dark Matter Halos with the Galaxy Center and the Supermassive Black Hole. Astrophys. J. 2015, 800, 124.

7. Bhattacharyya, S.; Biswas, P.; Patra, M.J. A leading-order comparison between fluid-gravity and membrane-gravity dualities. J. High Energy Phys. 2019, 2019, 22. doi:10.1007/JHEP05(2019)022.

8. Bredberg, I.; Keeler, C.; Lysov, V.; Strominger, A. From Navier-Stokes to Einstein. J. High Energ. Phys. 2012, 2012, 146. doi:10.1007/JHEP07(2012)146.

9. Jiménez, E.; Recalde, N.; Chacón, E. Extraction of the Proton and Electron Radii from Characteristic Atomic Lines and Entropy Principles. Entropy 2017, 19, 293. doi:10.3390/e19070293.

10. Jimenez, E. Nuclear Viscosity Obtained With the Solution of the Navier-Stokes Equations. IJMER 2018, 8, 1-16.

11. Jimenez, E.; Moya-Alvarez, D.; Alvarez-Samaniego, W.; Alvarez-Samaniego, B.; Jimenez, E.; Recalde, N. First Observation of an X-Ray Beam Following a New Geodesic When Gravitational Waves Deform Space-Time. J. Math. Res. 2019, 11, 2.

(C) 2019 by the authors. Licensee MDPI, Basel, Switzerland. This article is an open access article distributed under the terms and conditions of the Creative Commons Attribution (CC BY) license (http:/ / creativecommons.org/licenses/by/4.0/). 